\title{
On Three Theorems of Lees for Numerical Treatment of Semilinear Two-Point Boundary Value Problems
}

\author{
Tetsuro YAmamoto* and Shin'ichi Oishi ${ }^{\dagger}$ \\ School of Science and Engineering \\ Waseda University \\ Okubo 3-4-1, Shinjuku-ku, Tokyo 169-8555 \\ Japan \\ *E-mail: tetsuro.yamamoto@aoni.waseda.jp \\ †E-mail: oishi@waseda.jp
}

Received December 20, 2005

Revised July 18, 2006

\begin{abstract}
This paper is concerned with semilinear tow-point boundary value problems of the form $-\left(p(x) u^{\prime}\right)^{\prime}+f(x, u)=0, a \leq x \leq b, \alpha_{0} u(a)-\alpha_{1} u^{\prime}(a)=\alpha, \beta_{0} u(b)+\beta_{1} u^{\prime}(b)=\beta, \alpha_{i} \geq 0$, $\beta_{i} \geq 0, i=0,1, \alpha_{0}+\alpha_{1}>0, \beta_{0}+\beta_{1}>0, \alpha_{0}+\beta_{0}>0$. Under the assumption inf $f_{u}>-\lambda_{1}$, where $\lambda_{1}$ is the smallest eigenvalue of $\mathscr{L} u=-\left(p u^{\prime}\right)^{\prime}$ with the boundary conditions, unique existence theorems of solution for the continuous problem and a discretized system with not necessarily uniform nodes are given as well as error estimates. The results generalize three theorems of Lees for $u^{\prime \prime}=f(x, u), 0 \leq x \leq 1, u(0)=\alpha, u(1)=\beta$.
\end{abstract}

Key words: tow-point boundary value problems, discretization, existence of solution, error estimates, theorems of Lees

\section{Introduction}

We will be concerned with a mathematical theory for numerical treatment of semilinear boundary value problem

$$
\begin{aligned}
& -\frac{d}{d x}\left(p(x) \frac{d u}{d x}\right)+f(x, u)=0, \quad a \leq x \leq b, \\
& B_{1}(u)=\alpha_{0} u(a)-\alpha_{1} u^{\prime}(a)=\alpha, \\
& B_{2}(u)=\beta_{0} u(b)+\beta_{1} u^{\prime}(b)=\beta,
\end{aligned}
$$

where $p(x) \in C^{1}[a, b], p(x)>0, f(x, u) \in C([a, b] \times \mathbb{R})$ and $\alpha_{i}, \beta_{i}, i=0,1$ are constants which satisfy

$$
\begin{aligned}
& \alpha_{0} \geq 0, \quad \alpha_{1} \geq 0, \quad \alpha_{0}+\alpha_{1}>0, \\
& \beta_{0} \geq 0, \quad \beta_{1} \geq 0, \quad \beta_{0}+\beta_{1}>0,
\end{aligned}
$$

and

$$
\alpha_{0}+\beta_{0}>0
$$

Let $\mathscr{L} u=-(d / d x)(p(x)(d u / d x))$ and put $\mathscr{D}=\left\{u \in C^{2}[a, b] \mid B_{1}(u)=\right.$ $\left.B_{2}(u)=0\right\}$. Then, as is easily verified, the Green function for $(\mathscr{L}, \mathscr{D})$ exists 
under the conditions (1.4)-(1.6). It is known that if $f_{u}=\partial f / \partial u$ exists, is continuous on $[a, b] \times \mathbb{R}$ and $f_{u} \geq 0$, then the problem has a unique solution $u \in C^{2}[a, b]$ (cf. [8]; Remark 2.1). To find a numerical solution, we discretize (1.1)-(1.3) at not necessarily uniform nodes

$$
\Delta: a=x_{0}<x_{1}<\cdots<x_{n}<x_{n+1}=b,
$$

and put

$$
h_{i}=x_{i}-x_{i-1}, \quad h=\max _{i} h_{i}
$$

The discretized system we consider here is

$$
H A \boldsymbol{U}+\tilde{\boldsymbol{f}}(\boldsymbol{U})=\mathbf{0}
$$

where, if $\alpha_{1} \beta_{1} \neq 0$, then

$$
\begin{aligned}
& H=\left(\begin{array}{llll}
\omega_{0}^{-1} & & & \\
& \omega_{1}^{1} & & \\
& & \ddots & \\
& & & \omega_{n+1}^{-1}
\end{array}\right), \quad \omega_{i}= \begin{cases}\frac{1}{2} h_{1} & (i=0) \\
\frac{1}{2}\left(h_{i}+h_{j+1}\right) & (1 \leq i \leq n) \\
\frac{1}{2} h_{n+1} & (i=n+1),\end{cases} \\
& A=\left(\begin{array}{cccc}
a_{0}+a_{1} & -a_{1} & & \\
-a_{1} & a_{1}+a_{2} & -a_{2} & \\
\ddots & \ddots & \ddots & \\
& & -a_{n+1} & a_{n+1}+a_{n+2}
\end{array}\right) \\
& a_{i}= \begin{cases}\frac{\alpha_{0}}{\alpha_{1}} p(a) & (i=0) \\
\left(\int_{x_{i-1}}^{x_{i}} \frac{d t}{p(t)}\right)^{-1} & (1 \leq i \leq n+1) \\
\frac{\beta_{0}}{\beta_{1}} p(b) & (i=n+2),\end{cases} \\
& \boldsymbol{U}=\left(U_{0}, U_{1}, \ldots, U_{n+1}\right)^{t} \\
& \boldsymbol{f}(\boldsymbol{U})=\left(f\left(x_{0}, U_{0}\right), \ldots, f\left(x_{n+1}, U_{n+1}\right)\right)^{t} \\
& \widetilde{\boldsymbol{f}}(\boldsymbol{U})=\boldsymbol{f}(\boldsymbol{U})-\left(\frac{2}{h_{1}} \cdot \frac{\alpha}{\alpha_{1}} p(a), 0, \ldots, 0, \frac{2}{h_{n+1}} \cdot \frac{\beta}{\beta_{1}} p(b)\right)^{t} .
\end{aligned}
$$

If $\alpha_{1} \beta_{1}=0$, then (1.2) or (1.3) reduces to the Dirichlet condition $u(a)=0$ or $u(b)=0$ so that a modification of (1.8) is necessary. Namely, if $\alpha_{1}=0$ and $\beta_{1} \neq 0$, 
then $\alpha_{0} \neq 0$ and we replace $H, A, \boldsymbol{U}, \boldsymbol{f}$, and $\widetilde{\boldsymbol{f}}$ by

$$
\begin{aligned}
& H=\operatorname{diag}\left(\omega_{1}^{-1}, \omega_{2}^{-1}, \ldots, \omega_{n+1}^{-1}\right), \\
& A=\left(\begin{array}{cccc}
a_{1}+a_{2} & -a_{2} & & \\
-a_{2} & a_{2}+a_{3} & -a_{3} & \\
\ddots & \ddots & \ddots & \\
& & -a_{n+1} & a_{n+1}+a_{n+2}
\end{array}\right) \\
& \boldsymbol{U}=\left(U_{1}, U_{2}, \ldots, U_{n+1}\right)^{t}, \\
& \boldsymbol{f}(\boldsymbol{U})=\left(f\left(x_{1}, U_{1}\right), f\left(x_{2}, U_{2}\right), \ldots, f\left(x_{x+1}, U_{n+1}\right)\right)^{t}
\end{aligned}
$$

and

$$
\tilde{\boldsymbol{f}}(\boldsymbol{U})=\boldsymbol{f}(\boldsymbol{U})-\left(\frac{2}{h_{1}+h_{2}} \frac{\alpha}{\alpha_{0}} a_{1}, 0, \ldots, 0, \frac{2}{h_{n+1}} \cdot \frac{\beta}{\beta_{1}} p(b)\right)^{t} .
$$

If $a_{1} \neq 0$ and $\beta_{1}=0$, then $\beta_{0} \neq 0$ and $H, A, \boldsymbol{U}, \boldsymbol{f}$ and $\tilde{\boldsymbol{f}}$ in (1.8) are replaced by

$$
\begin{aligned}
& H=\operatorname{diag}\left(\omega_{0}^{-1}, \omega_{1}^{-1}, \ldots, \omega_{n}^{-1}\right),
\end{aligned}
$$

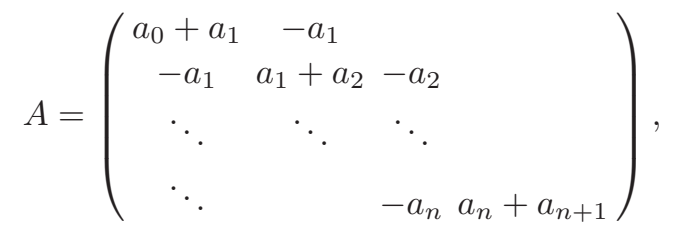

$$
\begin{aligned}
& \boldsymbol{U}=\left(U_{0}, U_{1}, \ldots, U_{n}\right)^{t}, \\
& \boldsymbol{f}(\boldsymbol{U})=\left(f_{0}\left(x_{0}, U_{0}\right), f\left(x_{1}, U_{1}\right), \ldots, f\left(x_{n}, U_{n}\right)\right)^{t}
\end{aligned}
$$

and

$$
\tilde{\boldsymbol{f}}(\boldsymbol{U})=\boldsymbol{f}(\boldsymbol{U})-\left(\frac{2}{h_{1}} \frac{\alpha}{\alpha_{1}} p(a), 0 \ldots, 0, \frac{2}{h_{n}+h_{n+1}} \frac{\beta}{\beta_{0}} a_{n+1}\right)^{t} .
$$

Furthermore, if $\alpha_{1}=\beta_{1}=0$, then $\alpha_{0}, \beta_{0} \neq 0$ and $H, A, \boldsymbol{U}, \boldsymbol{f}$ and $\tilde{\boldsymbol{f}}$ are replaced by

$$
\begin{aligned}
& H=\operatorname{diag}\left(\omega_{1}^{-1}, \omega_{2}^{-1}, \ldots, \omega_{n}^{-1}\right), \\
& A=\left(\begin{array}{cccc}
a_{1}+a_{2} & -a_{2} & & \\
-a_{2} & a_{2}+a_{3} & -a_{3} & \\
\ddots & \ddots & \ddots & \\
& & -a_{n} & a_{n}+a_{n+1}
\end{array}\right), \\
& \boldsymbol{U}=\left(U_{1}, \ldots, U_{n}\right)^{t}, \\
& \boldsymbol{f}(\boldsymbol{U})=\left(f\left(x_{1}, U_{1}\right), \ldots, f\left(x_{n}, U_{n}\right)\right)^{t}
\end{aligned}
$$

and

$$
\widetilde{\boldsymbol{f}}(\boldsymbol{U})=\boldsymbol{f}(\boldsymbol{U})-\left(\frac{2}{h_{1}+h_{2}} \frac{\alpha}{\alpha_{0}}, 0, \ldots, 0, \frac{2}{h_{n}+h_{n+1}} \frac{\beta}{\beta_{0}} a_{n+1}\right)^{t}
$$


It should be remarked here that, in any case, we have $A^{-1}=\left(G\left(x_{i}, x_{j}\right)\right)$ (cf. [8]), where $G(x, \xi)$ denotes the Green function for $(\mathscr{L}, \mathscr{D})$ with $\mathscr{L}=$ $-(d / d x)(p(d / d x)[])$.

Observe also that, if the nodes are uniform, i.e., $x_{i}=i h, h=(b-a) /(n+1)$, $p(x)=1$ and the boundary conditions are of Dirichlet's type $u(a)=\alpha$ and $u(b)=\beta$, then (1.8) reduces to a system of $n$ equations

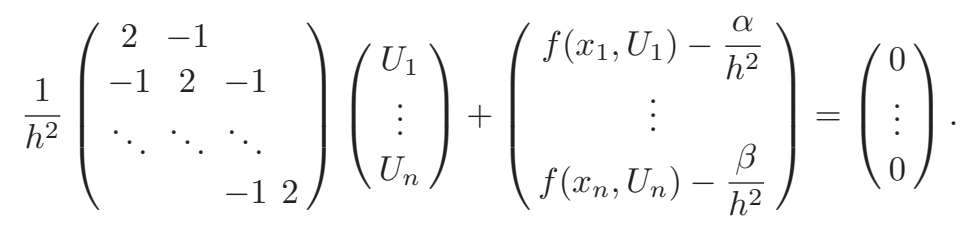

In [4], M. Lees considered the problem

$$
\begin{aligned}
& u^{\prime \prime}=f(x, u), \quad x \in E=[0,1], \\
& u(0)=\alpha, \quad u(1)=\beta
\end{aligned}
$$

and proved the following three theorems:

THEOREM 1.1. If $f_{u}$ exists, is continuous on $E \times \mathbb{R}$ and satisfies

$$
\inf _{E \times \mathbb{R}} f_{u}=-\eta>-\pi^{2},
$$

then the problem (1.11), (1.12) has a unique solution $u \in C^{2}[E]$.

TheOrem 1.2. Assume that $u \in C^{4}[E]$. If $f$ satisfies (1.13) and $h$ is sufficiently small, i.e., if $h \leq h_{0}$, where $h_{0}$ is a constant satisfying

$$
\eta<\pi^{2}\left[1-\frac{h_{0}^{2}}{12} \pi^{2}\right],
$$

then (1.10) has a unique solution $\boldsymbol{U}=\left(U_{1}, \ldots, U_{n}\right)^{t} \in \mathbb{R}^{n}$.

THEOREM 1.3. Let

$$
\|U\|_{D}=\sqrt{h \sum_{j=1}^{n+1}\left(\frac{U_{j}-U_{j-1}}{h}\right)^{2}},
$$

where $U_{0}=\alpha$ and $U_{n+1}=\beta$. Then, under the assumption of Theorem 1.2,

$$
\|\boldsymbol{u}-\boldsymbol{U}\|_{\infty} \leq \frac{1}{2}\|\boldsymbol{u}-\boldsymbol{U}\|_{D} \leq \frac{1}{12} K\left(h_{0}\right) h^{2}\left\|u^{(4)}\right\|_{E},
$$

where $\boldsymbol{u}=\left(u\left(x_{1}\right), \ldots, u\left(x_{n}\right)\right)^{t}, K\left(h_{0}\right)$ is a constant and

$$
\left\|u^{(4)}\right\|_{E}=\max _{x \in E}\left|u^{(4)}(x)\right| \text {. }
$$

The purpose of this paper is to generalize these result to the problem (1.1)-(1.6) and its discretized system (1.8). 


\section{Existence of Solution for (1.1)-(1.6)}

Observing that the number $\pi^{2}$ in (1.13) is the smallest eigenvalue for the operator $-\left(d^{2} / d x^{2}\right):\left\{u \in C^{2}[0,1] \mid u(0)=u(1)=0\right\} \rightarrow C[0,1]$, we generalize Theorem 1.1 on the basis of the following three lemmas.

Lemma 2.1. Let $\mathscr{L} u=-(d / d x)(p(d u / d x))$ and $\mathscr{D}=\left\{u \in C^{2}[a, b] \mid B_{1}(u)=\right.$ $\left.B_{2}(u)=0\right\}$. We denote by $\lambda_{1}$ the smallest eigenvalue of $(\mathscr{L}, \mathscr{D})$. Then $\lambda_{1}$ is positive and

$$
(\mathscr{L} u, u) \geq \lambda_{1}\|u\|^{2} \quad \forall u \in \mathscr{D}
$$

where $\|\cdot\|$ denote the $L_{2}$ norm.

Proof. The proof is straightforward since $(\mathscr{L}, \mathscr{D})$ has a complete system of orthonrmal eigenfunctinos in $L_{2}[a, b]$.

Lemma 2.2. Let $r(x), g(x) \in C[a, b]$ and

$$
\min _{a \leq x \leq b} r(x)=-\eta>-\lambda_{1}
$$

where $\lambda_{1}$ is as defined by Lemma 2.1. Then the boundary value problem

$$
\begin{aligned}
& \mathscr{L} u+r(x) u=g(x), \quad a \leq x \leq b \\
& u \in \mathscr{D}
\end{aligned}
$$

has a unique solution and

$$
\|u\| \leq \frac{\|g\|}{\lambda_{1}-\eta} .
$$

Proof. Let $\mathscr{L} u+r(x) u=0, u \in \mathscr{D}$. Then

$$
\begin{aligned}
0 & =(\mathscr{L} u, u)+(r u, u) \\
& \geq \lambda_{1}(u, u)+(r u, u)=\left(\left(\lambda_{1}+r\right) u, u\right) \geq\left(\lambda_{1}-\eta\right)(u, u) .
\end{aligned}
$$

Since $\lambda_{1}-\eta>0$, we obtain $\|u\|=0$. Hence, $(\mathscr{L}+r I, \mathscr{D})$, where $I$ is the identity, is injective and the problem

$$
\mathscr{L} u+r u=g, \quad u \in \mathscr{D}
$$

has a unique solution. We then have

$$
\left(\lambda_{1}-\eta\right)\|u\|^{2} \leq(\mathscr{L} u, u)+(r u, u)=(g, u) \leq\|g\| \cdot\|u\| .
$$

If $\|u\|>0$, then (2.3) implies

$$
\left(\lambda_{1}-\eta\right)\|u\| \leq\|g\|
$$


or

$$
\|u\| \leq \frac{\|g\|}{\lambda_{1}-\eta} .
$$

This inequality holds for $\|u\|=0$, too.

Lemma 2.3. Let $G(x, \xi)$ be the Green function for $(\mathscr{L}, \mathscr{D})$, where $\mathscr{L}$ and $\mathscr{D}$ are defined in Lemma 2.1. Then

$$
\begin{aligned}
& G(x, \xi) \\
& =\left\{\begin{array}{l}
\frac{1}{p(a) p(b) \delta}\left(\alpha_{1}+\alpha_{0} p(a) \int_{a}^{x} \frac{d t}{p(t)}\right)\left(\beta_{1}+\beta_{0} p(b) \int_{\xi}^{b} \frac{d t}{p(t)}\right) \quad(x \leq \xi) \\
\frac{1}{p(a) p(b) \delta}\left(\alpha_{1}+\alpha_{0} p(a) \int_{a}^{\xi} \frac{d t}{p(t)}\right)\left(\beta_{1}+\beta_{0} p(b) \int_{x}^{b} \frac{d t}{p(t)}\right) \quad(x \geq \xi)
\end{array}\right. \\
& \leq G(x, x)
\end{aligned}
$$

where

$$
\delta=\alpha_{0}\left(\beta_{0} \int_{a}^{b} \frac{d t}{p(t)}+\frac{\beta_{1}}{p(b)}\right)+\frac{\alpha_{1} \beta_{0}}{p(a)}>0 .
$$

Proof. See [8].

We are now in a position to prove the following:

THEOREM 2.1. If $f(x, u)$ satisfies

$$
\inf _{[a, b] \times \mathbb{R}} f_{u}(x, u)=-\eta>-\lambda_{1},
$$

where $\lambda_{1}$ is defined in Lemma 2.1, then the problem (1.1)-(1.6) has a unique solution $u \in C^{2}[a, b]$.

Proof. Without loss of generality, we may assume $\alpha=\beta=0$ (cf. [8]).

(i) Existence. Putting

$$
r(x ; u)=\int_{0}^{1} f_{u}(x ; \theta u) d \theta,
$$

we have

$$
f(x, u)=f_{0}(x)+r(x ; u) u,
$$

where $f_{0}(x)=f(x, 0)$. Then, by Lemma 2.2 , given $u \in C[a, b]$, the linear boundary value problem

$$
\mathscr{L} w+r(x ; u) w=-f_{0}(x), \quad w \in \mathscr{D}
$$

has a unique solution $w \in C^{2}[a, b]$, which satisfies

$$
\|w\| \leq \frac{\left\|f_{0}\right\|}{\lambda_{1}-\eta} \equiv \delta_{0} \quad \text { (say) }
$$


by Lemma 2.2. Furthermore, we have from (2.6)

$$
\mathscr{L} w=-f_{0}(x)-r(x ; u) w
$$

and

$$
w(x)=-\int_{a}^{b} G(x, \xi)\left\{f_{0}(\xi)+r(\xi ; u(\xi)) w(\xi)\right\} d \xi
$$

so that, using Lemma 2.3, we obtain

$$
\begin{aligned}
\frac{d w(x)}{d x}= & -\int_{a}^{x} \frac{\partial G(x, \xi)}{\partial x}\left\{f_{0}(\xi)+r(\xi ; u(\xi)) w(\xi)\right\} d \xi \\
& -\int_{x}^{b} \frac{\partial G(x, \xi)}{\partial x}\left\{f_{0}(\xi)+r(\xi ; u(\xi)) w(\xi)\right\} d \xi \\
= & -\int_{a}^{x} \frac{1}{\delta}\left(-\frac{\beta_{0}}{p(x)}\right)\left(\frac{\alpha}{p(a)}+\alpha_{0} \int_{a}^{\xi} \frac{d t}{p(t)}\right)\left\{f_{0}(\xi)+r(\xi ; u(\xi)) w(\xi)\right\} d \xi \\
& -\int_{x}^{b} \frac{1}{\delta}\left(\frac{\alpha_{0}}{p(x)}\right)\left(\frac{\beta_{1}}{p(b)}+\beta_{0} \int_{\xi}^{b} \frac{d t}{p(t)}\right)\left\{f_{0}(\xi)+r(\xi ; u(\xi)) w(\xi)\right\} d \xi
\end{aligned}
$$

where $\delta$ is as defined in (2.4).

Observing that

$$
\delta=\beta_{0}\left(\frac{\alpha_{1}}{p(a)}+\alpha_{0} \int_{a}^{b} \frac{d t}{p(t)}\right)+\frac{\alpha_{0} \beta_{1}}{p(b)} \geq \beta_{0}\left(\frac{\alpha_{1}}{p(a)}+\alpha_{0} \int_{a}^{\xi} \frac{d t}{p(t)}\right)
$$

and similary

$$
\delta \geq \alpha_{0}\left(\frac{\beta_{1}}{p(b)}+\beta_{0} \int_{\xi}^{b} \frac{d t}{p(t)}\right)
$$

we have

$$
\begin{aligned}
\left|\frac{d w(x)}{d x}\right| \leq & \int_{a}^{x} \frac{1}{p(x)}\left(\left|f_{0}(\xi)\right|+|r(\xi ; u(\xi))| \cdot|w(\xi)|\right) d \xi \\
& +\int_{x}^{b} \frac{1}{p(x)}\left(\left|f_{0}(\xi)\right|+|r(\xi ; u(\xi))| \cdot|w(\xi)|\right) d \xi \\
= & \int_{a}^{b} \frac{1}{p(x)}\left(\left|f_{0}(\xi)\right|+|r(\xi ; u(\xi))| \cdot|w(\xi)|\right) d \xi \\
\leq & \frac{1}{p_{*}} \int_{a}^{b}\left(\left\|f_{0}\right\|_{[a, b]}+|r(\xi ; u(\xi))| \cdot\|w\|_{[a, b]}\right) d \xi
\end{aligned}
$$

where $p_{*}=\min _{a \leq x \leq b} p(x)>0$ and $\|\cdot\|_{[a, b]}$ denotes the maximum norm in $[a, b]$ :

$$
\left\|f_{0}\right\|_{[a, b]}=\max _{x \in[a, b]}\left|f_{0}(x)\right|, \quad\|w\|_{[a, b]}=\max _{x \in[a, b]}|w(x)|, \quad \text { etc. }
$$


Hence, if $u \in C^{2}[a, b]$ and $\|u\|_{[a, b]} \leq \delta_{0}$, then, putting

$$
\widetilde{K}=\sup _{[a, b] \times\left[-\delta_{0}, \delta_{0}\right]}\left|f_{u}(x, u)\right|
$$

we have

$$
\begin{aligned}
\left\|w^{\prime}\right\|_{[a, b]} & \leq \frac{1}{p_{*}} \int_{a}^{b}\left(\left\|f_{0}\right\|_{[a, b]}+\widetilde{K} \delta_{0}\right) d \xi \\
& =\frac{1}{p_{*}}\left(\left\|f_{0}\right\|_{[a, b]}+\widetilde{K} \delta_{0}\right)(b-a) \equiv \delta_{1} \quad \text { (say). }
\end{aligned}
$$

Consider a Banach space $X=C^{1}[a, b]$ equipped with the norm $\|u\|_{C^{1}}=\|u\|_{[a, b]}+$ $\left\|u^{\prime}\right\|_{[a, b]}$ for $u \in X$ and put

$$
S=\left\{u \in X \mid\|u\|_{[a, b]} \leq \delta_{0},\left\|u^{\prime}\right\|_{[a, b]} \leq \delta_{1}, B_{1}(u)=B_{2}(u)=0\right\} .
$$

Then $S$ is a bounded and closed convex set in $X$. The map $T: S \rightarrow S \cap C^{2}[a, b] \subset S$ defined by $T u=w, u \in S$, is then continuous and it can be shown by Ascoli-Arzela's theorem that $T(S)$ is relatively compact in $X$ (cf. [8]). Hence, Schauder's theorem implies that $T$ has a fixed point $u \in S$. It is clear that $u=T u$ is a solution of (1.1)-(1.6).

(ii) Uniqueness. Let $u$ and $v$ be two solutions of the problem and $\operatorname{set} \varphi=$ $u-v$. Then

$$
f(x, u)-f(x, v)=r(x ; u, v) \varphi
$$

where

$$
r(x ; u, v)=\int_{0}^{1} f_{u}(x, v+\theta(u-v)) d \theta .
$$

Therefore

$$
\begin{aligned}
& \mathscr{L} \varphi+r(x ; u, v) \varphi=0, \quad a \leq x \leq b \\
& \varphi \in \mathscr{D}
\end{aligned}
$$

where

$$
r(x ; u, v) \geq-\eta>-\lambda_{1} .
$$

Hence Lemma 2.2 applies to conclude that $\varphi \equiv 0$.

REMARK 2.1. If $\alpha_{1} \beta_{1} \neq 0$ and $\alpha=\beta=0$, then the existence of solution for the problem (1.1)-(1.6) follows from the following result which is the onedimensional version of Theorem 2.3.1 in Sattinger [5]. (Also see Amann [1], [2])

THEOREM 2.2. Let $\varphi(x)$ and $\psi(x)$ be upper and lower solutions for the problem (1.1)-(1.6) with $\alpha=\beta=0$ :

$$
\begin{array}{llll}
\mathscr{L} \varphi+f(x, \varphi) \geq 0 & (a \leq x \leq b), & B_{1}(\varphi) \geq 0, & B_{2}(\varphi) \geq 0 \\
\mathscr{L} \psi+f(x, \psi) \leq 0 & (a \leq x \leq b), & B_{1}(\psi) \leq 0, & B_{2}(\psi) \leq 0,
\end{array}
$$


where $\mathscr{L}$ is as defined in Lemma 2.1. If $\psi \leq \varphi$ in $[a, b]$, then there exists a solution $u$ for (1.1)-(1.6) with $\psi \leq u \leq \varphi$.

In fact, if we put $F(x, u)=f(x, u)+\lambda_{1} u$ where $\lambda_{1}$ is defined in Lemma 2.1, then

$$
F_{u}(x, u)=f_{u}(x, u)+\lambda_{1} \geq \lambda_{1}-\eta>0 .
$$

Hence, at each $x$ fixed, $F$ is monotonically increasing in $u$ and $F(x,-\infty)=-\infty$, $F(x,+\infty)=+\infty, x \in[a, b]$, so that, by the implicit function theorem, there exists a unique $\Phi(x) \in C[a, b]$ satisfying $F(x, \Phi(x))=0$. Take a positive constant $m$ with $-m \leq \Phi(x) \leq m \forall x \in[a, b]$ and an eigenfunction $v(x)$ corresponding to the eigenvalue $\lambda_{1}$ for $(\mathscr{L}, \mathscr{D})$. Then $v(x)>0$ in $[a, b]$ since $\alpha_{1} \beta_{1} \neq 0$.

Furthermore, letting

$$
\gamma=\min _{a \leq x \leq b} v(x)>0
$$

we have

$$
-\frac{m}{\gamma} v(x) \leq-m \leq \Phi(x) \leq m \leq \frac{m}{\gamma} v(x) \quad \forall x \in[a, b]
$$

and it can be shown that $\varphi(x)=(m / \gamma) v(x)$ and $\psi(x)=-(m / \gamma) v(x)$ are upper and lower solutions with $\psi(x) \leq \varphi(x)$ in $[a, b]$.

In fact, we have

$$
\begin{aligned}
\mathscr{L} \varphi+f(x, \varphi) & =\mathscr{L} \varphi-\lambda_{1} \varphi+F(x, \varphi) \\
& =F(x, \varphi) \\
& \geq F(x, \Phi(x))=0
\end{aligned}
$$

and

$$
B_{1}(\varphi)=B_{2}(\varphi)=0
$$

Similarly

$$
\mathscr{L} \psi+f(x, \psi)=F(x, \psi) \leq F(x, \Phi(x))=0
$$

and

$$
B_{1}(\psi)=B_{2}(\psi)=0
$$

Hence, by Theorem 2.2, the problem (1.1)-(1.6) has a solution $u$ with $\psi \leq u \leq \varphi$.

\section{Existence of Solution for the Discretized System}

In this section, we shall show that (1.8) has a unique solution $\boldsymbol{U} \in \mathbb{R}^{n+2}$ for sufficiently small $h$. Before doing this, we prepare several lemmas. In the following, we assume $\alpha_{1} \beta_{1} \neq 0$ without loss of generality. 
LEMMA 3.1. Let $\widehat{U}(x)$ be the piecewise linear interpolant for the $n+2$ points $\left(x_{i}, U_{i}\right), i=0,1,2, \ldots, n+1$. Then

$$
\frac{1}{3}\left(\boldsymbol{U}, H^{-1} \boldsymbol{U}\right) \leq\|\widehat{U}\|^{2} \leq\left(\boldsymbol{U}, H^{-1} \boldsymbol{U}\right)
$$

Proof. The trapezoidal rule for numerical integration implies that if $\varphi \in$ $C^{2}\left[x_{j-1}, x_{j}\right], 1 \leq j \leq n+1$, then there exists $\xi_{j} \in\left(x_{j-1}, x_{j}\right)$ for each $j$ such that

$$
\int_{x_{j-1}}^{x_{j}} \varphi(x) d x=\frac{h_{j}}{2}\left(\varphi\left(x_{j}\right)+\varphi\left(x_{j-1}\right)\right)-\frac{h_{j}^{3}}{12} \varphi^{\prime \prime}\left(\xi_{j}\right) .
$$

Hence

$$
\begin{aligned}
\int_{a}^{b} \varphi(x) d x & =\sum_{j=1}^{n+1} \frac{h_{j}}{2}\left(\varphi\left(x_{j}\right)+\varphi\left(x_{j-1}\right)\right)-\frac{1}{12} \sum_{j=1}^{n+1} h_{j}^{3} \varphi^{\prime \prime}\left(\xi_{j}\right) \\
& =\sum_{j=0}^{n+1} \omega_{j} \varphi\left(x_{j}\right)-\frac{1}{12} \sum_{j=1}^{n+1} h_{j}^{3} \varphi^{\prime \prime}\left(\xi_{j}\right),
\end{aligned}
$$

or

$$
\sum_{j=0}^{n+1} \omega_{j} \varphi\left(x_{j}\right)=\int_{a}^{b} \varphi(x) d x+\frac{1}{12} \sum_{j=1}^{n+1} h_{j}^{3} \varphi^{\prime \prime}\left(\xi_{j}\right)
$$

An application of this formula to $\varphi(x)=\{\widehat{U}(x)\}^{2}$ yields

$$
\begin{aligned}
\left(\boldsymbol{U}, H^{-1} \boldsymbol{U}\right) & =\sum_{j=0}^{n+1} \omega_{j} U_{j}^{2}=\sum_{j=0}^{n+1} \omega_{j}\left\{\widehat{U}\left(x_{j}\right)\right\}^{2} \\
& =\int_{a}^{b} \widehat{U}(x)^{2} d x+\frac{1}{6} \sum_{j=1}^{n+1} h_{j}^{3}\left(\frac{U_{j}-U_{j-1}}{h_{j}}\right)^{2} \\
& =\|\widehat{U}\|^{2}+\frac{1}{6} \sum_{j=1}^{n+1} h_{j}\left(U_{j}-U_{j-1}\right)^{2} \\
& \geq\|\widehat{U}\|^{2},
\end{aligned}
$$

where we have used the fact that

$$
\widehat{U}(x)=\frac{U_{j}-U_{j-1}}{h_{j}}\left(x-x_{j-1}\right)+U_{j-1}, \quad x \in\left[x_{j-1}, x_{j}\right]
$$

and

$$
\frac{d^{2}}{d x^{2}}\left\{\widehat{U}(x)^{2}\right\}=\frac{2\left(U_{j}-U_{j-1}\right)^{2}}{h_{j}^{2}} \quad(\text { constant }), \quad x \in\left[x_{j-1}, x_{j}\right]
$$


On the other hand

$$
\begin{aligned}
\int_{a}^{b} \widehat{U}(x)^{2} d x & =\sum_{j=1}^{n+1} \int_{x_{j-1}}^{x_{j}}\left\{\frac{U_{j}-U_{j-1}}{h_{j}}\left(x-x_{j-1}\right)+U_{j-1}\right\}^{2} d x \\
& =\sum_{j=1}^{n+1}\left\{\left(U_{j}-U_{j-1}\right)^{2} \frac{h_{j}}{3}+2\left(U_{j}-U_{j-1}\right) U_{j-1} \frac{h_{j}}{2}+U_{j-1}^{2} h_{j}\right\} \\
& =\frac{1}{3} \sum_{j=1}^{n+1} h_{j}\left(U_{j}^{2}+U_{j} U_{j-1}+U_{j-1}^{2}\right) \\
& \geq \frac{1}{3} \sum_{j=1}^{n+1} \frac{h_{j}}{2}\left(U_{j}^{2}+U_{j-1}^{2}\right) \\
& =\frac{1}{3} \sum_{j=0}^{n+1} \omega_{j} U_{j}^{2}=\frac{1}{3}\left(\boldsymbol{U}, H^{-1} \boldsymbol{U}\right)
\end{aligned}
$$

This proves Lemma 3.1.

LEMMA 3.2. The following inequalities hold.

(i) $\sum_{j=1}^{n+1} h_{j}\left|U_{j}-U_{j-1}\right|^{2} \leq 4\left(\boldsymbol{U}, H^{-1} \boldsymbol{U}\right)$

(ii) $\sum_{j=1}^{n+1} h_{j}\left|U_{j}-U_{j-1}\right| \leq 2 \sqrt{b-a} \sqrt{\left(\boldsymbol{U}, H^{-1} \boldsymbol{U}\right)}$

(iii) $\sum_{j=1}^{n+1} h_{j}\left|U_{j-1}\right|^{2} \leq 2\left(\boldsymbol{U}, H^{-1} \boldsymbol{U}\right)$

(iv) $\sum_{j=1}^{n+1} h_{j}\left|U_{j-1}\right| \leq \sqrt{2(b-a)} \sqrt{\left(\boldsymbol{U}, H^{-1} \boldsymbol{U}\right)}$

(v) $\sum_{j=1}^{n+1} h_{j} \max \left(\left|U_{j-1}\right|^{2},\left|U_{j}\right|^{2}\right) \leq 2\left(\boldsymbol{U}, H^{-1} \boldsymbol{U}\right)$

(vi) $\sum_{j=1}^{n+1} h_{j} \max \left(\left|U_{j-1}\right|,\left|U_{j}\right|\right) \leq 2 \sqrt{b-a} \sqrt{\left(\boldsymbol{U}, H^{-1} \boldsymbol{U}\right)}$

Proof. For examples, we have

$$
\begin{aligned}
& \sum_{j=1}^{n+1} h_{j}\left|U_{j}-U_{j-1}\right|^{2} \leq 2 \sum_{j=1}^{n+1} h_{j}\left(U_{j}^{2}+U_{j-1}^{2}\right)=4\left(\boldsymbol{U}, H^{-1} \boldsymbol{U}\right) \\
& \sum_{j=1}^{n+1} h_{j}\left|U_{j}-U_{j-1}\right| \leq \sqrt{\sum_{j=1}^{n+1}\left(\sqrt{h_{j}}\right)^{2} \sum_{J=1}^{n+1}\left(\sqrt{h_{j}}\right)^{2}\left|U_{j}-U_{j-1}\right|^{2}} \\
& \leq \sqrt{(b-a) \cdot 4\left(\boldsymbol{U}, H^{-1} \boldsymbol{U}\right)} \\
&=2 \sqrt{b-a} \sqrt{\left(\boldsymbol{U}, H^{-1} \boldsymbol{U}\right)}, \quad \text { etc. }
\end{aligned}
$$

Lemma 3.3. $\int_{a}^{b}|\widehat{U}(x)| d x \leq(1+\sqrt{2}) \sqrt{b-a} \sqrt{\left(\boldsymbol{U}, H^{-1} \boldsymbol{U}\right)}$.

Proof. We have from Lemma 3.2

$$
\begin{aligned}
\int_{a}^{b}|\widehat{U}(x)| d x & =\sum_{i=1}^{n+1} \int_{x_{i-1}}^{x_{i}}|\widehat{U}(x)| d x \\
& \leq \sum_{i=1}^{n+1} \int_{x_{i-1}}^{x_{i}}\left\{\frac{\left|U_{i}-U_{i-1}\right|}{h_{i}}\left(x-x_{i-1}\right)+\left|U_{i-1}\right|\right\} d x
\end{aligned}
$$




$$
\begin{aligned}
& \leq \sum_{i=1}^{n+1}\left(\frac{\left|U_{i}-U_{i-1}\right|}{2}+\left|U_{i-1}\right|\right) h_{i} \\
& \leq(1+\sqrt{2}) \sqrt{b-a} \sqrt{\left(\boldsymbol{U}, H^{-1} \boldsymbol{U}\right)} .
\end{aligned}
$$

Since the Green function $G(x, \xi)$ belongs to $C^{2}$ class in the regions $\Omega_{1}=\{(x, \xi) \mid$ $a \leq x \leq \xi \leq b\}$ and $\Omega_{2}=\{(x, \xi) \mid a \leq \xi \leq x \leq b\}$, there exists a constant $M>0$ such that

$$
\left|\frac{\partial^{k} G(x, \xi)}{\partial x^{k}}\right| \leq M \quad(0 \leq k \leq 2)
$$

in $\Omega_{1}$ or $\Omega_{2}$.

LEMMA 3.4. Let $\left(\boldsymbol{U}, H^{-1} \boldsymbol{U}\right)=1$. Then

$$
\sum_{j=0}^{n+1} G\left(x_{i}, x_{j}\right) \omega_{j} U_{j}=\int_{a}^{b} G\left(x_{i}, \xi\right) \widehat{U}(\xi) d \xi+\varepsilon_{i}, \quad 0 \leq i \leq n+1,
$$

where

$$
\left|\varepsilon_{i}\right| \leq \varepsilon \equiv \frac{M h}{6}(2+h) \sqrt{b-a}=O(h) .
$$

Proof. Let $\widehat{U}(x)$ be as defined in Lemma 3.1 and put

$$
\varphi_{i}(\xi)=G\left(x_{i}, \xi\right) \widehat{U}(\xi) .
$$

Then

$$
\begin{aligned}
\int_{a}^{b} G\left(x_{i}, \xi\right) \widehat{U}(\xi) d \xi & =\sum_{j=1}^{n+1} \int_{x_{j-1}}^{x_{j}} G\left(x_{i}, \xi\right) \widehat{U}(\xi) d \xi \\
& =\sum_{j=1}^{n+1}\left[\frac{h_{j}}{2}\left\{\varphi_{i}\left(x_{j-1}\right)+\varphi_{i}\left(x_{j}\right)\right\}-\frac{h_{j}^{3}}{12} \varphi_{i}^{\prime \prime}\left(\xi_{j}\right)\right] \\
& \quad\left(x_{j-1} \leq \xi_{j} \leq x_{j}\right) \\
& =\sum_{j=0}^{n+1} \omega_{j} \varphi_{i}\left(x_{j}\right)-\varepsilon_{i},
\end{aligned}
$$

where $\varepsilon_{i}=(1 / 12) \sum_{j=1}^{n+1} h_{j}^{3} \varphi_{i}^{\prime \prime}\left(\xi_{j}\right)$.

If $x_{j-1}<\xi<x_{j}$, then

$$
\varphi^{\prime \prime}(\xi)=\frac{\partial^{2} G\left(x_{i}, \xi\right)}{\partial \xi^{2}} \widehat{U}(\xi)+2 \frac{\partial G\left(x_{i}, \xi\right)}{\partial \xi} \widehat{U}^{\prime}(\xi)
$$

and

$$
\begin{aligned}
\left|\varphi^{\prime \prime}(\xi)\right| & \leq M|\widehat{U}(\xi)|+2 M \frac{\left|U_{j}-U_{j-1}\right|}{h_{j}} \\
& \leq M \max \left(\left|U_{j-1}\right|,\left|U_{j}\right|\right)+2 M \frac{\left|U_{j}-U_{j-1}\right|}{h_{j}} .
\end{aligned}
$$


Hence we have

$$
\frac{h_{j}^{3}}{12}\left|\varphi^{\prime \prime}\left(\xi_{j}\right)\right| \leq \frac{M}{12} h_{j}^{3} \max \left(\left|U_{j-1}\right|,\left|U_{j}\right|\right)+\frac{M}{6} h_{j}^{2}\left|U_{j}-U_{j-1}\right|
$$

and, by Lemma 3.2,

$$
\begin{aligned}
\left|\varepsilon_{i}\right| & \leq \frac{1}{12} \sum_{j=1}^{n+1} h_{j}^{3}\left|\varphi^{\prime \prime}\left(\xi_{j}\right)\right| \\
& \leq \frac{M}{12} \sum_{j=1}^{n+1} h_{j}^{3} \max \left(\left|U_{j-1}\right|,\left|U_{j}\right|\right)+\frac{1}{6} \sum_{j=1}^{n+1} h_{j}^{2}\left|U_{j}-U_{j-1}\right| \\
& \leq \frac{M h^{2}}{12} \sum_{j=1}^{n+1} h_{j} \max \left(\left|U_{j-1}\right|,\left|U_{j}\right|\right)+\frac{M h}{6} \sum_{j=1}^{n+1} h_{j}\left|U_{j}-U_{j-1}\right| \\
& \leq \frac{M h^{2}}{6} \sqrt{b-a} \sqrt{\left(\boldsymbol{U}, H^{-1} \boldsymbol{U}\right)}+\frac{M h}{6} 2 \sqrt{b-a} \sqrt{\left(\boldsymbol{U}, H^{-1} \boldsymbol{U}\right)} \\
& =\frac{M h}{6}(h+2) \sqrt{b-a} \sqrt{\left(\boldsymbol{U}, H^{-1} \boldsymbol{U}\right)} \\
& =\frac{M h}{6}(h+2) \sqrt{b-a}=\varepsilon .
\end{aligned}
$$

LEMMA 3.5. Let $\left(\boldsymbol{U}, H^{-1} \boldsymbol{U}\right)=1$. Then

$$
\begin{aligned}
& \int_{a}^{b} \int_{a}^{b} G(x, \xi) \widehat{U}(\xi) d \xi \widehat{U}(x) d x \\
& =\sum_{i=0}^{n+1} \sum_{j=0}^{n+1} G\left(x_{i}, x_{j}\right)\left(\omega_{i} U_{i}\right)\left(\omega_{j} U_{j}\right)+O(h) .
\end{aligned}
$$

Proof. Let

$$
\psi(x)=\int_{a}^{b} G(x, \xi) \widehat{U}(\xi) d \xi \widehat{U}(x) .
$$

Then

$$
\begin{aligned}
& \int_{a}^{b} \int_{a}^{b} G(x, \xi) \widehat{U}(\xi) d \xi \widehat{U}(x) d x \\
& =\sum_{i=1}^{n+1} \int_{x_{i-1}}^{x_{i}} \psi(x) d x \\
& =\sum_{i=1}^{n+1}\left\{\frac{h_{i}}{2}\left[\psi\left(x_{i-1}\right)+\psi\left(x_{i}\right)\right]-\frac{h_{i}^{3}}{12} \psi^{\prime \prime}\left(\eta_{i}\right)\right\}, \quad x_{i-1}<\eta_{i}<x_{i} .
\end{aligned}
$$


If $x_{i-1}<x<x_{i}$, then

$$
\begin{aligned}
\psi^{\prime \prime}(x)= & \left(\int_{a}^{b} \frac{\partial^{2} G(x, \xi)}{\partial x^{2}} \widehat{U}(\xi) d \xi-\frac{\widehat{U}(x)}{p(x)}\right) \widehat{U}(x) \\
& +2 \int_{a}^{b} \frac{\partial G(x, \xi)}{\partial x} \widehat{U}(\xi) d \xi \cdot \widehat{U}^{\prime}(x)
\end{aligned}
$$

and

$$
\begin{aligned}
\left|\psi^{\prime \prime}(x)\right| \leq & \left(M \int_{a}^{b}|\widehat{U}(\xi)| d \xi+\frac{1}{p_{*}} \max \left(\left|U_{i-1}\right|,\left|U_{i}\right|\right)\right) \max \left(\left|U_{i-1}\right|,\left|U_{i}\right|\right) \\
& +2 M \int_{a}^{b}|\widehat{U}(\xi)| d \xi \cdot \frac{\left|U_{i}-U_{i-1}\right|}{h_{i}}
\end{aligned}
$$

Therefore,

$$
\begin{aligned}
& \frac{1}{12} \sum_{i=1}^{n+1} h_{i}^{3}\left|\varphi^{\prime \prime}\left(\eta_{i}\right)\right| \\
& \leq\left(\frac{M}{12} \int_{a}^{b}|\widehat{U}(\xi)| d \xi\right) \sum_{i=1}^{n+1} h_{i}^{3} \max \left(\left|U_{i-1}\right|,\left|U_{i}\right|\right) \\
& \quad+\frac{1}{12 p_{*}} \sum_{i=1}^{n+1} h_{i}^{3} \max \left(\left|U_{i-1}\right|^{2},\left|U_{i}\right|^{2}\right) \\
& \quad+\frac{M}{6} \int_{a}^{b}|\widehat{U}(\xi)| d \xi \sum_{i=1}^{n+1} h_{i}^{2}\left|U_{i}-U_{i-1}\right| \\
& \leq \frac{M}{6} \sqrt{b-a} h^{2} \int_{a}^{b}|\widehat{U}(\xi)| d \xi+\frac{h^{2}}{6 p_{*}} \\
& \quad+\frac{M h}{3} \sqrt{b-a} \int_{a}^{b}|\widehat{U}(\xi)| d \xi \\
& \leq \frac{M}{6}(1+\sqrt{2})(b-a) h^{2}+\frac{h^{2}}{6 p_{*}}+\frac{M}{3}(1+\sqrt{2})(b-a) h \\
&= \widetilde{\varepsilon} \quad(\text { say }) .
\end{aligned}
$$

By Lemma 3.4, we have

$$
\psi\left(x_{i}\right)=\left(\sum_{j=0}^{n+1} G\left(x_{i}, x_{j}\right) \omega_{j} U_{j}-\varepsilon_{i}\right) U_{i} .
$$

We obtain from (3.1) that

$$
\int_{a}^{b} \int_{a}^{b} G(x, \xi) \widehat{U}(\xi) d \xi \widehat{U}(x) d x
$$




$$
\begin{aligned}
& =\sum_{i=1}^{n+1} \frac{h_{i}}{2}\left[\psi\left(x_{i-1}\right)+\psi\left(x_{i}\right)\right]-\frac{1}{12} \sum_{i=1}^{n+1} h_{i}^{3} \psi^{\prime \prime}\left(\eta_{i}\right) \\
& =\sum_{i=0}^{n+1} \psi\left(x_{i}\right) \omega_{i}-\frac{1}{12} \sum_{i=1}^{n+1} h_{i}^{3} \psi^{\prime \prime}\left(\eta_{i}\right) \\
& =\sum_{i=0}^{n+1}\left(\sum_{j=0}^{n+1} G\left(x_{i}, x_{j}\right) \omega_{j} U_{j}-\varepsilon_{i}\right) U_{i} \omega_{i}-\frac{1}{12} \sum_{i=1}^{n+1} h_{i}^{3} \psi^{\prime \prime}\left(\eta_{i}\right) \\
& =\sum_{i=0}^{n+1} \sum_{j=0}^{n+1} G\left(x_{i}, x_{j}\right)\left(\omega_{j} U_{j}\right)\left(\omega_{i} U_{i}\right)+\sigma,
\end{aligned}
$$

where

$$
\sigma=-\sum_{i=0}^{n+1} \varepsilon_{i}\left(U_{i} \omega_{i}\right)-\frac{1}{12} \sum_{i=1}^{n+1} h_{i}^{3} \psi^{\prime \prime}\left(\eta_{i}\right) .
$$

We thus obtain from Lemma 3.4 and (3.2)

$$
\begin{aligned}
|\sigma| & \leq \sum_{i=0}^{n+1}\left|\varepsilon_{i}\right| \cdot\left|U_{i}\right| \omega_{i}+\widetilde{\varepsilon} \\
& \leq \varepsilon \sum_{i=0}^{n+1} \omega_{i}\left|U_{i}\right|+\widetilde{\varepsilon} \\
& =\varepsilon \sum_{i=1}^{n+1} \frac{h_{i}}{2}\left(\left|U_{i}\right|+\left|U_{i-1}\right|\right)+\widetilde{\varepsilon} \\
& \leq \varepsilon \sqrt{b-a}\left(\boldsymbol{U}^{-1}, H^{-1} \boldsymbol{U}\right)+\widetilde{\varepsilon} \\
& =\varepsilon \sqrt{b-a}+\widetilde{\varepsilon} \\
& =O(h) \sqrt{b-a}+O(h)=O(h) .
\end{aligned}
$$

Lemma 3.6. For sufficiently small $h$, the matrix $H A-\eta I$ is an M-matrix, hence nonsingular.

Proof. Since $A$ is an irreducibly diagonally dominant $L$-matrix and symmetric, $A$ is a positive definite $M$-matrix. Then, for any $\boldsymbol{U}, \boldsymbol{V} \in \mathbb{R}^{n+2}$, we have

$$
\begin{aligned}
(\boldsymbol{U}, \boldsymbol{V})^{2} & =\left(\sqrt{A} \boldsymbol{U}, \sqrt{A}^{-1} \boldsymbol{V}\right)^{2} \\
& \leq(\sqrt{A} \boldsymbol{U}, \sqrt{A} \boldsymbol{U})\left(\sqrt{A}^{-1} \boldsymbol{V}, \sqrt{A}^{-1} \boldsymbol{V}\right) \\
& =(A \boldsymbol{U}, \boldsymbol{U})\left(A^{-1} \boldsymbol{V}, \boldsymbol{V}\right) .
\end{aligned}
$$

Let $\boldsymbol{W} \in \mathbb{R}^{n+2}$ and $\boldsymbol{W} \neq \mathbf{0}$. Then $c=\left(\boldsymbol{W}, H^{-1} \boldsymbol{W}\right)>0$. We put $\boldsymbol{U}=(1 / \sqrt{c}) \boldsymbol{W}$. Then we have $\left(\boldsymbol{U}, H^{-1} \boldsymbol{U}\right)=1$. 
We then have from (3.3), Lemma 3.1 and Lemma 3.5

$$
\begin{aligned}
\left(\left(A-\eta H^{-1}\right) \boldsymbol{U}, \boldsymbol{U}\right) & =(A \boldsymbol{U}, \boldsymbol{U})-\eta\left(\boldsymbol{U}, H^{-1} \boldsymbol{U}\right) \\
& \geq \frac{\left(\boldsymbol{U}, H^{-1} \boldsymbol{U}\right)^{2}}{\left(A^{-1} H^{-1} \boldsymbol{U}, H^{-1} \boldsymbol{U}\right)}-\eta\left(\boldsymbol{U}, H^{-1} \boldsymbol{U}\right) \\
& =\left[\frac{\left(\operatorname{Put} \boldsymbol{V}=H^{-1} \boldsymbol{U} \text { in }(3.3)\right)}{\sum_{i=0}^{n+1} \sum_{j=0}^{n+1} G\left(x_{i}, x_{j}\right)\left(\omega_{i} U_{i}\right)\left(\omega_{j} U_{j}\right)}-\eta\right]\left(\boldsymbol{U}, H^{-1} \boldsymbol{U}\right) \\
& \geq \frac{\|\widehat{U}\|^{2}}{\int_{a}^{b} \int_{a}^{b} G(x, \xi) \widehat{U}(\xi) d \xi \widehat{U}(x) d x+O(h)}-\eta \\
& \geq \frac{\|\widehat{U}\|^{2}}{\left(1 / \lambda_{1}\right)\|\widehat{U}\|^{2}+O(h)}-\eta \\
& =\left(\lambda_{1}-\eta\right)+\frac{O(h)}{\|\widehat{U}\|^{2}} .
\end{aligned}
$$

By Lemma 3.1, we have $\|\widehat{U}\|=O(1)$ and

$$
\lambda_{1}-\eta+\frac{O(h)}{\|\widehat{U}\|^{2}}>0
$$

for sufficiently small $h>0$ and $\left(\left(A-\eta H^{-1}\right) \boldsymbol{W}, \boldsymbol{W}\right)>0$ for any $\boldsymbol{W} \neq \mathbf{0}$. Consequently the symmetric matrix $B=A-\eta H^{-1}$ is then positive definite and eigenvalues are all positive. Since $B$ is a $Z$-matrix, this means that $B$ as well as $H B=H A-\eta I$ is an $M$-matrix (cf. [7]).

We are now in a position to prove the following:

TheOREM 3.1. Under the assumption of Theorem 2.1, the discretized system (1.8) has a unique solution if $h$ is sufficiently small.

Proof. We again assume $\alpha=\beta=0$, without loss of generality.

(i) Uniqueness. Let $\boldsymbol{U}, \boldsymbol{V} \in \mathbb{R}^{n+2}$ be two solutions of (1.8) and put $\boldsymbol{W}=$ $\boldsymbol{U}-\boldsymbol{V}=\left(W_{0}, W_{1}, \ldots, W_{n+1}\right)^{t}$. Then $\boldsymbol{W}$ satisfies the system of $(n+2)$ equations

$$
(H A+D) \boldsymbol{W}=\mathbf{0},
$$

where

$$
D=\operatorname{diag}\left(d_{0}, d_{1}, \ldots, d_{n+1}\right),
$$

with

$$
d_{i}=\int_{0}^{1} f_{u}\left(x_{i}, V_{i}+\theta\left(U_{i}-V_{i}\right)\right) d \theta, \quad 0 \leq i \leq n+1
$$


By Lemma 3.6, we have for sufficiently small $h$

$$
\left(\left(A+H^{-1} D\right) \boldsymbol{U}, \boldsymbol{U}\right) \geq\left(\left(A-\eta H^{-1}\right) \boldsymbol{U}, \boldsymbol{U}\right)>0 \quad \forall \boldsymbol{U}(\neq \mathbf{0}) \in \mathbb{R}^{n+2},
$$

and $H A+D$ is nonsingular. We thus obtain $\boldsymbol{W}=\mathbf{0}$, which means the uniqueness of the solution.

(ii) Existence. We write (1.8) as

$$
H A \boldsymbol{U}+Z \boldsymbol{U}=-\tilde{\boldsymbol{f}}(\mathbf{0})
$$

where

$$
Z=Z(\boldsymbol{U})=\operatorname{diag}\left(\zeta_{0}, \zeta_{1}, \ldots, \zeta_{n+1}\right)
$$

with

$$
\zeta_{i}=\int_{0}^{1} f_{u}\left(x_{i}, \theta U_{i}\right) d \theta, \quad 0 \leq i \leq n+1,
$$

and

$$
\begin{aligned}
\widetilde{\boldsymbol{f}}(\mathbf{0}) & =\left(f\left(x_{0}, 0\right), f\left(x_{1}, 0\right), \ldots, f\left(x_{n+1}, 0\right)\right)^{t} \\
& =\left(f_{0}\left(x_{0}\right), f_{0}\left(x_{1}\right), \ldots, f_{0}\left(x_{n+1}\right)\right)^{t}
\end{aligned}
$$

with $f_{0}(x)=f(x, 0)$.

Since $Z \geq-\eta I$ ( $I$ is the $(n+2) \times(n+2)$ identity), given $\boldsymbol{U} \in \mathbb{R}^{n+2}$, the system of linear equations

$$
(H A+Z) \boldsymbol{W}=-\tilde{\boldsymbol{f}}(\mathbf{0})
$$

has a unique solution

$$
\begin{aligned}
\boldsymbol{W} & =-(H A+Z)^{-1} \tilde{\boldsymbol{f}}(\mathbf{0}) \\
& =-\left(A+H^{-1} Z\right)^{-1} H^{-1} \widetilde{\boldsymbol{f}}(\mathbf{0})
\end{aligned}
$$

where $A+H^{-1} Z$ is again a symmetric $M$-matrix. Let $\varphi \in C^{2}[a, b]$ be the unique solution of the problem

$$
-\frac{d}{d x}\left(p(x) \frac{d u}{d x}\right)-\eta u=2, \quad u \in \mathscr{D},
$$

whose existence is guaranteed by Theorem 2.1. Let

$$
\tau=(H A-\eta I) \varphi-2 e,
$$

where

$$
\boldsymbol{\varphi}=\left(\varphi\left(x_{0}\right), \varphi\left(x_{1}\right), \ldots, \varphi\left(x_{n+1}\right)\right)^{t} \quad \text { and } \quad \boldsymbol{e}=(1,1, \ldots, 1)^{t} \in \mathbb{R}^{n+2} .
$$


Then a simple computation based upon the usual Taylor expansion for $p(x) \in$ $C^{1}[a, b]$ and $\varphi(x) \in C^{2}[a, b]$ yields

$$
\|\boldsymbol{\tau}\|_{\infty}=o(1) \rightarrow 0
$$

as $h \rightarrow 0$, where we apply the midpoint rule to estimate the integrals $a_{i}=$ $\int_{x_{i-1}}^{x_{i}}(d t / p(t)), 1 \leq i \leq n+1$ and employ the fact that $p^{\prime}$ and $\varphi^{\prime \prime}$ are uniformly continuous in the interval $[a, b]$. Hence, for sufficiently small $h$,

$$
(H A-\eta I) \varphi=2 \boldsymbol{e}+\boldsymbol{\tau} \geq \boldsymbol{e}
$$

or

$$
(H A-\eta I)^{-1} e \leq \varphi .
$$

We denote by $|\boldsymbol{W}|$ the vector $\left(\left|W_{0}\right|,\left|W_{1}\right|, \ldots,\left|W_{n+1}\right|\right)^{t}$.

Then we have from (3.6)

$$
\begin{aligned}
|\boldsymbol{W}| & \leq\left(A+H^{-1} Z\right)^{-1} H^{-1}|\tilde{\boldsymbol{f}}(\mathbf{0})| \\
& \leq\left(A-\eta H^{-1}\right)^{-1} H^{-1}\|\tilde{\boldsymbol{f}}(\mathbf{0})\|_{\infty} \boldsymbol{e} \\
& =\|\tilde{\boldsymbol{f}}(\mathbf{0})\|_{\infty}(H A-\eta I)^{-1} \boldsymbol{e} \\
& \leq\|\tilde{\boldsymbol{f}}(\mathbf{0})\|_{\infty} \boldsymbol{\varphi}
\end{aligned}
$$

and

$$
\|\boldsymbol{W}\|_{\infty} \leq\|\widetilde{\boldsymbol{f}}(\mathbf{0})\|_{\infty}\|\boldsymbol{\varphi}\|_{\infty} \leq\left\|f_{0}\right\|_{[a, b]}\|\varphi\|_{[a, b]}=C \quad \text { (say) }
$$

since we have assumed $\alpha=\beta=0$ and $\tilde{\boldsymbol{f}}(\mathbf{0})=\left(f_{0}\left(x_{0}\right), f_{0}\left(x_{1}\right), \ldots, f_{0}\left(x_{n+1}\right)\right)^{t}$. Hence we put

$$
S=\left\{\boldsymbol{U} \in \mathbb{R}^{n+2} \mid\|\boldsymbol{U}\|_{\infty} \leq C\right\}
$$

and define a map $T: S \rightarrow S$ by $T \boldsymbol{U}=\boldsymbol{W}, \boldsymbol{U} \in S$. Then $T$ is continuous. In fact, we have for $\boldsymbol{U}, \widehat{U} \in S$

$$
\begin{aligned}
T \boldsymbol{U}-T \widehat{\boldsymbol{U}} & =-\left\{(H A+Z)^{-1}-(H A+\widehat{Z})^{-1}\right\} \tilde{\boldsymbol{f}}(\mathbf{0}) \\
& =(H A+\widehat{Z})^{-1}(Z-\widehat{Z})(H A+Z)^{-1} \widetilde{\boldsymbol{f}}(\mathbf{0})
\end{aligned}
$$

where $Z=Z(\boldsymbol{U})$ and $\widehat{Z}=Z(\widehat{\boldsymbol{U}})$. It now follows from (3.8) and (3.9) that

$$
\|T \boldsymbol{U}-T \widehat{\boldsymbol{U}}\|_{\infty} \leq\|Z-\widehat{Z}\|_{\infty}\|\varphi\|_{[a, b]} C \rightarrow 0
$$

as $\|\boldsymbol{U}-\widehat{\boldsymbol{U}}\|_{\infty} \rightarrow 0$, since $\|Z-\widehat{Z}\|_{\infty} \rightarrow 0$ as $\|\boldsymbol{U}-\widehat{\boldsymbol{U}}\|_{\infty} \rightarrow 0$ because of the uniform continuity of $f_{u}(x, u)$ in $[a, b] \times[-C, C]$.

Consequently, we conclude, by Brouwer's theorem, that $T$ has a fixed point $U$ in $S$, which is a solution of (1.8). 


\section{Error Estimates}

We still keep the assumption $\alpha_{1} \beta_{1} \neq 0$ without loss of generality.

Let $u=u(x)$ be the solution of the continuous problem (1.1)-(1.6) and $\boldsymbol{u}=$ $\left(u\left(x_{0}\right), u\left(x_{1}\right), \ldots, u\left(x_{n+1}\right)\right)^{t}$. We put

$$
\boldsymbol{\tau}=H A \boldsymbol{u}+\tilde{\boldsymbol{f}}(\boldsymbol{u})=\left(\tau_{0}, \tau_{1}, \ldots, \tau_{n+1}\right)^{t} .
$$

Then we have from (1.8)

$$
H A(\boldsymbol{u}-\boldsymbol{U})+\tilde{\boldsymbol{f}}(\boldsymbol{u})-\tilde{\boldsymbol{f}}(\boldsymbol{U})=\boldsymbol{\tau}
$$

or

$$
(H A+\widetilde{D})(\boldsymbol{u}-\boldsymbol{U})=\boldsymbol{\tau}
$$

where $\widetilde{D}=\operatorname{diag}\left(\widetilde{d}_{0}, \widetilde{d}_{1}, \ldots, \widetilde{d}_{n+1}\right)^{t}$ with $\widetilde{d}_{i}=\int_{0}^{1} f_{u}\left(x_{i}, U_{i}+\theta\left(u_{i}-U_{i}\right)\right) d \theta$. As is shown in the end of the proof of Lemma 3.6, $H A-\eta I$ is an $M$-matrix and

$$
H A+\widetilde{D} \geq H A-\eta I
$$

Hence, $H A+\widetilde{D}$ is an $M$-matrix and

$$
0<(H A+\widetilde{D})^{-1} \leq(H A-\eta I)^{-1}
$$

since $H A+\widetilde{D}$ is an irreducible $Z$-matrix and $(H A+\widetilde{D})^{-1}$ is a positive matrix $([7])$.

It now follows from (4.1) and (4.2) that

$$
\boldsymbol{u}-\boldsymbol{U}=(H A+\widetilde{D})^{-1} \boldsymbol{\tau} .
$$

As is easily seen, we have

$$
\|\boldsymbol{\tau}\|_{\infty}= \begin{cases}o(1) & \left(\text { if } u \in C^{2}[a, b]\right) \\ O(h) & \left(\text { if } u \in C^{2,1}[a, b], p \in C^{1,1}[a, b]\right)\end{cases}
$$

and

$$
\begin{aligned}
|\boldsymbol{u}-\boldsymbol{U}| & \leq(H A+\widetilde{D})^{-1}|\boldsymbol{\tau}| \leq\|\boldsymbol{\tau}\|_{\infty}(H A+\widetilde{D})^{-1} \boldsymbol{e} \\
& \leq\|\boldsymbol{\tau}\|_{\infty}(H A-\eta)^{-1} \boldsymbol{e} \leq\|\boldsymbol{\tau}\|_{\infty} \boldsymbol{\varphi} \quad \text { by }(3.7)
\end{aligned}
$$

Hence

$$
\|\boldsymbol{u}-\boldsymbol{U}\|_{\infty}= \begin{cases}o(1) & \left(u \in C^{2}[a, b]\right) \\ O(h) & \left(u \in C^{2,1}[a, b]\right)\end{cases}
$$


Furthermore, if $p \in C^{2,1}[a, b]$ and $u \in C^{3,1}[a, b]$, then it can be shown (see [8] pp. 52-56) that

$$
\tau_{i}= \begin{cases}O\left(h_{1}\right) & (i=0) \\ \frac{2}{h_{i}+h_{i+1}}\left(s_{i+(1 / 2)} h_{i+1}^{2}-s_{i-(1 / 2)} h_{i}^{2}\right) u_{i}^{\prime}-\left(h_{i+1}-h_{i}\right) \kappa_{i}+O\left(h^{2}\right) & (1 \leq i \leq n) \\ O\left(h_{n+1}\right) & (i=n+1)\end{cases}
$$

where

$$
\begin{aligned}
& s(x)=\frac{1}{24}\left(\frac{1}{p}\right)^{\prime \prime} p^{2}, \\
& \kappa(x)=\frac{1}{12}\left\{3 p^{\prime \prime}(x) u^{\prime}(x)+6 p^{\prime}(x) u^{\prime \prime}(x)+4 p(x) u^{\prime \prime \prime}(x)\right\} \\
& s_{i+(1 / 2)}=s\left(x_{i}+\frac{1}{2} h_{i+1}\right), \\
& s_{i-(1 / 2)}=s\left(x_{i}-\frac{1}{2} h_{i}\right)
\end{aligned}
$$

and

$$
\kappa_{i}=\kappa\left(x_{i}\right)
$$

We have from (4.3)

$$
\begin{aligned}
\boldsymbol{u}-\boldsymbol{U} & =(H A+\widetilde{D})^{-1} \boldsymbol{\tau} \\
& =\left[(H A)^{-1}-(H A+\widetilde{D})^{-1} \widetilde{D}(H A)^{-1}\right] \boldsymbol{\tau} \\
& =A^{-1} H^{-1} \boldsymbol{\tau}-(H A+\widetilde{D})^{-1} \widetilde{D}\left(A^{-1} H^{-1} \boldsymbol{\tau}\right)
\end{aligned}
$$

and

$$
\begin{aligned}
|\boldsymbol{u}-\boldsymbol{U}| & \leq\left|A^{-1} H^{-1} \boldsymbol{\tau}\right|+(H A+\widetilde{D})^{-1}|\widetilde{D}|\left|A^{-1} H^{-1} \boldsymbol{\tau}\right| \\
& \leq\left|A^{-1} H^{-1} \boldsymbol{\tau}\right|+(H A-\eta I)^{-1}|\widetilde{D}|\left|A^{-1} H^{-1} \boldsymbol{\tau}\right|
\end{aligned}
$$

Since $\|\boldsymbol{U}-\boldsymbol{u}\|_{\infty} \rightarrow 0$ as $h \rightarrow 0$ by (4.4), $\|\boldsymbol{u}\|_{\infty} \leq\|u\|_{[a, b]} \leq \delta_{0}$ (cf. the proof of Theorem 2.1) and, for any $\theta \in[0,1]$,

$$
\begin{aligned}
\|\boldsymbol{U}+\theta(\boldsymbol{u}-\boldsymbol{U})\|_{\infty} & =\|\boldsymbol{u}+(1-\theta)(\boldsymbol{U}-\boldsymbol{u})\|_{\infty} \\
& \leq\|\boldsymbol{u}\|_{\infty}+(1-\theta)\|\boldsymbol{U}-\boldsymbol{u}\|_{\infty} \\
& \leq\|\boldsymbol{u}\|_{\infty}+\|\boldsymbol{U}-\boldsymbol{u}\|_{\infty}
\end{aligned}
$$

we may assume that $\|\boldsymbol{U}+\theta(\boldsymbol{u}-\boldsymbol{U})\|_{\infty} \leq 2 \delta_{0} \forall \theta \in[0,1]$.

Then

$$
\left|\widetilde{d}_{i}\right| \leq \widehat{K}=\max _{[a, b] \times\left[-2 \delta_{0}, 2 \delta_{0}\right]}\left|f_{u}(x, u)\right|<+\infty \quad \forall i
$$


Therefore we obtain from (4.7) and (3.7)

$$
\begin{aligned}
\|\boldsymbol{u}-\boldsymbol{U}\|_{\infty} & \leq\left\|A^{-1} H^{-1} \boldsymbol{\tau}\right\|_{\infty}+\widehat{K}\left\|A^{-1} H^{-1} \boldsymbol{\tau}\right\|_{\infty}\|\varphi\|_{\infty} \\
& \leq\left(1+\widehat{K}\|\varphi\|_{[a, b]}\right)\left\|A^{-1} H^{-1} \boldsymbol{\tau}\right\|_{\infty} .
\end{aligned}
$$

If $p \in C^{2,1}[a, b]$ and $u \in C^{3,1}[a, b]$, then we have

$$
\left(A^{-1} H^{-1} \tau\right)_{i}=\sum_{j=0}^{n+1} G\left(x_{i}, x_{j}\right) \omega_{j} \tau_{j}
$$

and we can show

$$
\left\|A^{-1} H^{-1} \boldsymbol{\tau}\right\|_{\infty}=O\left(h^{2}\right)
$$

by noting that the functions $s(x)$ and $\kappa(x)$ defined by (4.5) and (4.6) are Lipschitz continuous in $[a, b]$ (cf. [8]). Hence, from (4.8) we have $\|\boldsymbol{u}-\boldsymbol{U}\|_{\infty}=O\left(h^{2}\right)$.

Summarizing we have the following result.

TheOREM 4.1. Under the assumption (2.5), we have

$$
\|\boldsymbol{u}-\boldsymbol{U}\|_{\infty}= \begin{cases}o(1) & \left(u \in C^{2}[a, b], p \in C^{1}[a, b]\right) \\ O(h) & \left(u \in C^{2,1}[a, b], p \in C^{1,1}[a, b]\right) \\ O\left(h^{2}\right) & \left(u \in C^{3,1}[a, b], p \in C^{2,1}[a, b]\right) .\end{cases}
$$

\section{Remark}

In (1.8), if we replace the integral $\int_{x_{i-1}}^{x_{i}}(d t / p(t))$ by the mid point formula $h_{i} / p\left(x_{i-(1 / 2)}\right)\left(x_{i-(1 / 2)}=(1 / 2)\left(x_{i}+x_{i-1}\right)\right)$ for each $i$, then the usual finite difference formula arises. We can also derive Theorems 3.1 and 4.1 in this case.

Acknowledgements. The authors are grateful to the referees for their helpful comments, by which this paper has been improved. Among others, constructions of upper and lower solutions described in Remark 2.1 are due to one of the referees.

\section{References}

[1] H. Amann, On the existence of positive solutions of nonlinear elliptic boundary value problems. Indiana Univ. Math. J., 21 (1971), 125-146.

[2] H. Amann, Fixed point equations and nonlinear eigenvalue problems in ordered Banach spaces. SIAM Review, 18 (1976), 620-709.

[ 3 ] H.B. Keller, Numerical Methods for Two-Point Boundary Value Problems. Blaisdell 1968.

[4] M. Lees, Discrete method for nonlinear two-point boundary value problems. Numerical Solution of Partial Differential Equations, J.H. Bramble, ed., Academic Press, 1966, 59-72.

[ 5 ] D.H. Sattinger, Topics in Stability and Bifurcation Theory. Lecture Notes in Mathematics, 309, Springer, 1973.

[ 6 ] A.N. Tikhonov and A.A. Samarskii, Homogeneous difference schemes of a high degree of accuracy on non-uniform nets. U.S.S.R. Comp. Math. \& Math. Physics, 1 (1961), 465-486.

[ 7 ] R.S. Varga, Matrix Iterative Analysis. Springer, 2000.

[8] T. Yamamoto and S. Oishi, A mathematical theory for numerical treatment of nonlinear two-point boundary value problems. Japan JIAM, 23 (2006), 31-62. 